\title{
Utilization of a Cloud-Based Diabetes Management Program for Insulin Initiation and Titration Enables Collaborative Decision Making Between Healthcare Providers and Patients
}

\author{
William C. Hsu, MD, ${ }^{1, *}$ Ka Hei Karen Lau, MS, RD, CDE, ${ }^{1, *}$ Ruyi Huang, MD, \\ Suzanne Ghiloni, RN, CDE, Hung Le, BS, Scott Gilroy, BS, \\ Martin Abrahamson, MD, and John Moore, MD, PhD ${ }^{4}$
}

\begin{abstract}
Background: Overseeing proper insulin initiation and titration remains a challenging task in diabetes care. Recent advances in mobile technology have enabled new models of collaborative care between patients and healthcare providers (HCPs). We hypothesized that the adoption of such technology could help individuals starting basal insulin achieve better glycemic control compared with standard clinical practice.

Materials and Methods: This was a 12 \pm 2 -week randomized controlled study with 40 individuals with type 2 diabetes who were starting basal insulin due to poor glycemic control. The control group $(n=20)$ received standard face-to-face care and phone follow-up as needed in a tertiary center, whereas the intervention group $(n=20)$ received care through the cloud-based diabetes management program where regular communications about glycemic control and insulin doses were conducted via patient self-tracking tools, shared decision-making interfaces, secure text messages, and virtual visits (audio, video, and shared screen control) instead of office visits. Results: By intention-to-treat analysis, the intervention group achieved a greater hemoglobin A1c decline compared with the control group $(3.2 \pm 1.5 \%$ vs. $2.0 \% \pm 2.0 \% ; P=0.048)$. The Diabetes Treatment Satisfaction Questionnaire showed a significant improvement in the intervention group compared with the control group (an increase of $10.1 \pm 11.7$ vs. $2.1 \pm 6.5$ points; $P=0.01$ ). HCPs spent less time with patients in the intervention group compared with those in the control group (65.9 min per subject vs. $81.6 \mathrm{~min}$ per subject). However, the intervention group required additional training time to use the mobile device.
\end{abstract}

Conclusions: Mobile health technology could be an effective tool in sharing data, enhancing communication, and improving glycemic control while enabling collaborative decision making in diabetes care.

\section{Introduction}

T ODAY'S MULTIDISCIPLINARY DIABETES CARE model is personnel intensive and is limited by access to healthcare providers (HCPs). Visits are increasingly short in duration.
Many patients are not adequately followed up until the next office visit. ${ }^{1}$ The outpatient-based face-to-face care model, which may be suitable for managing acute illnesses, is inadequate for managing chronic, noncommunicable diseases, which now account for the majority of deaths in developed

\footnotetext{
${ }^{1}$ Joslin Diabetes Center, Harvard Medical School, Boston, Massachusetts.

${ }^{2}$ Harvard T.H. Chan School of Public Health, Boston, Massachusetts; E-Da Hospital and I-Shou University, School of Medicine, Kaohsiung, Taiwan.

${ }^{3}$ University of Massachusetts Medical School, Worcester, Massachusetts.

${ }^{4}$ MIT Media Lab, Cambridge, Massachusetts.

*The first two authors contributed equally to this work.

This study is registered with ClinicalTrials.gov with clinical trial registration number NCT01698528.

(C) William C. Hsu et al., 2016; Published by Mary Ann Liebert, Inc. This Open Access article is distributed under the terms of the Creative Commons Attribution Noncommercial License (http://creativecommons.org/licenses/by-nc/4.0/) which permits any noncommercial use, distribution, and reproduction in any medium, provided the original author(s) and the source are credited.
} 
countries. $^{2}$ For many, living with diabetes is an overwhelmingly full-time job. Patients need advice on managing their glucose levels through the vicissitudes of life, like changes in food, physical activity, and stress, where most of the challenges occur in between medical visits. Taking care of chronic illnesses like diabetes requires a different model where patients can be empowered through self-management. ${ }^{3}$

One of the challenges that HCPs and patients face in managing type 2 diabetes is starting and titrating basal insulin, as an add-on to either metformin or multiple antihyperglycemic drugs. This has become a standard of treatment endorsed by the American Diabetes Association. ${ }^{3-5}$ Because numerous published studies have shown that treat to target by using basal insulin is effective in lowering hemoglobin A1c (HbA1c) levels, an indicator of blood glucose control over past 2-3 months, ${ }^{6-8}$ many clinical practices have adopted various algorithms for insulin titration for their patients. For instance, the PREDICTIVE 303 algorithm for insulin titration requires that the patient obtain daily fasting blood glucose measurements and increase or decrease 3 units of insulin every 3 days if the level is above or below a predetermined goal of achieving a fasting glucose level between 80 and $110 \mathrm{mg} / \mathrm{dL} .{ }^{9}$ However, there are many practical barriers to successfully initiating and titrating insulin to target. Even after 90 years since the discovery of insulin, inappropriate insulin use is still a leading cause for emergency hospitalizations. ${ }^{10}$

The emerging advances in mobile and Web-based technologies have fundamentally altered the patterns of information exchange in our society. These new technologies have the potential to provide real-time diabetes self-management tools, which include sharing of data and connecting HCPs with patients in between medical visits. More recently, new studies have supported the claims that the combination of behavioral mobile coaching with blood glucose data sharing via a telemedicine platform substantially reduced $\mathrm{HbAlc}$ levels, ${ }^{1-14}$ providing some evidence that mobile health applications can be effective in diabetes management.

In this study we developed a diabetes management program that was specifically designed to support individuals with type 2 diabetes working with HCPs to initiate and titrate basal insulin, guided by the PREDICTIVE 303 algorithm ${ }^{9}$ and hypoglycemia treatment guidelines. ${ }^{15,16}$ This clinical scenario was chosen for the study because basal insulin titration characteristically requires significant clinical support, which is time consuming. Insulin initiation and titration have also been studied extensively, so there are several metrics for comparison. ${ }^{6-9,17}$

The diabetes management program was specifically designed based on the model of cognitive apprenticeship that is informed by the situated learning theory. ${ }^{18}$ In this model the clinician coach models expert decision making to the patient by carefully articulating his or her decision-making process to the patient. Then the patient is encouraged to emulate the same decisionmaking process under the direct guidance of the clinician coach. The clinician coach gradually decreases support and eventually only observes for safety. The patient learns through active participation rather than through lecture/handouts, which is the basis of the situated learning theory. ${ }^{18}$ The goal of this approach was to help patients develop improved self-efficacy and more accurate management models for diabetes. In our study, a cloud-based diabetes management program was used to share data, communicate, and enable collaborative decision making between the patient and HCPs. We hypothesized that the use of such system with HCPs would help individuals starting basal insulin achieve better glycemic control compared with standard clinical practice at a tertiary diabetes clinic.

\section{Materials and Methods}

This was a randomized controlled study lasting $12 \pm 2$ weeks with staggered recruitment. This period was chosen because it is the minimal time required to assess a change in HbA1c level.

The recruitment took place in a tertiary diabetes center with care provided by teams of endocrinologists, nurse practitioners, and certified diabetes educators. Subjects with type 2 diabetes ( $\geq 18$ years of age with HbA1c levels of 9-14\%) who were being started on basal insulin therapy by their treating HCPs and had internet connectivity were eligible for inclusion in the study. Patients starting basal insulin were trained by a diabetes educator before actually commencing therapy. A member of the study staff assessed patient interest in the study after this diabetes educator meeting. Subjects with significant visual or hearing impairment, who were not proficient in English, who were pregnant or lactating, who had alcohol dependency, or who required multiple daily insulin injections were excluded.

Subjects interested in participating in the study went through a process of written informed consent to enroll in the study. An $\mathrm{HbA1c}$ test was obtained prior to starting basal insulin. After each subject completed a Diabetes Treatment Satisfaction Questionnaire (DTSQ) and the staff collected baseline clinical data, the study subject was randomly assigned to the intervention or the control group. This study was approved by the Institutional Review Boards at the Massachusetts Institute of Technology (MIT) and the Joslin Diabetes Center.

\section{Diabetes management program}

The diabetes management program was developed using the CollaboRhythm software platform designed at the MIT Media Lab, Cambridge, MA. The program supports the development of self-efficacy in diabetes care through selftracking tools, shared decision-making interfaces for subjects and HCPs, and streamlined communications tools (secure text messages and virtual visits).

Self-tracking in the program begins with the co-creation of a diabetes care plan between the subjects and HCPs. The plan can include any number of medications a day, which can be scheduled at specific times with flexible adherence windows. The plan is visualized for the patient on the tablet computer application in order to provide daily awareness and to allow self-tracking of medication adherence and blood glucose. (A wireless glucose meter [model D40b; ForaCare $^{\circledR}$, Moorpark, CA] is integrated into the program and automates the reporting of blood glucose.) All selftracking data across all subjects are synchronized in real time with the clinician coach's application (Fig. 1).

The shared decision-making interfaces include weekly charts to help the subjects and HCPs to see the correlation between medication adherence and blood glucose readings. The charts are paired with a visualization of the PREDICTIVE 303 protocol that is updated in real time based on the subject's most recent data. The three most recent blood glucose values are highlighted, the mean is automatically calculated and displayed with respect to decision-making thresholds, and the recommended dose change ( -3 units, no 
1.2. $\begin{aligned} & \text { Robert } \\ & \text { Brooks }\end{aligned}$

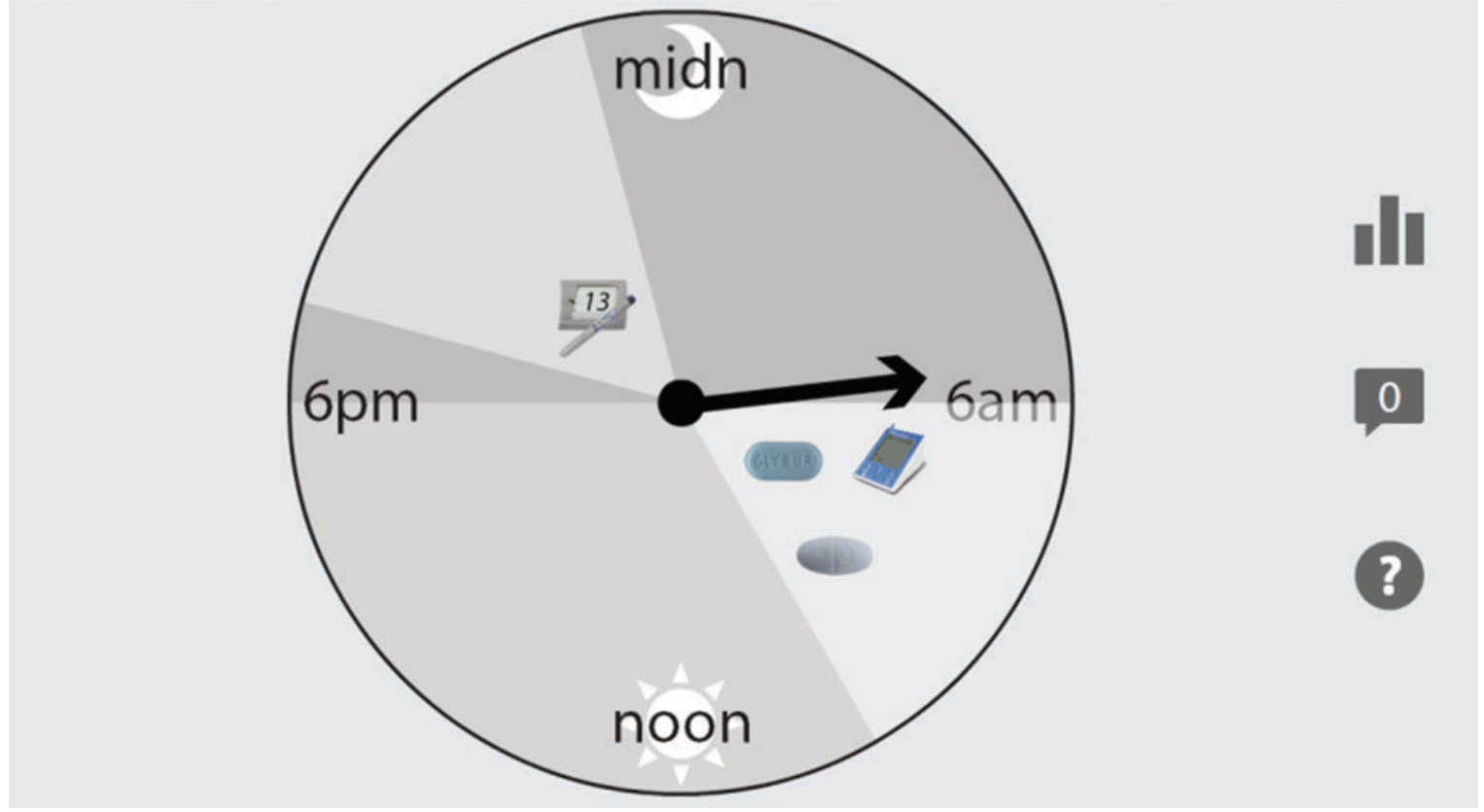

FIG. 1. Self-tracking visualization. The 24-h clock shows all of the subject's scheduled health actions. In this case the subject has three health actions scheduled between 6 a.m. and 10 a.m. (two pills and a blood glucose measurement) and one health action scheduled between 7 p.m. and 11 p.m. (an injection of 13 units of insulin). He can click on any of these health actions to see more information and to report adherence. Subjects can see and report their health actions even before they are due, which allows for proactive planning in their busy lives. The three buttons along the right side of the view are shortcuts to charts, messaging, and frequently asked questions. (The name and photograph used in this example do not belong to any study subject.)

change, or +3 units) based on the protocol is highlighted. The patient and the clinician coach are free to choose the desired change in insulin dose, and the interface emphasizes that other factors, such as medication adherence and diet and exercise, should be accounted for in the decision (Fig. 2).

The streamlined communication tools integrated into the application help facilitate timely learning and clinical support based on trends in data and decision-making events. The secure text messages have the advantage of efficiency, and the virtual visits (audio, video, and shared screen control) allow for much more in-depth co-exploration of the data and collaborative decision making. Typically virtual visits are used more frequently in the early stages by the subjects and HCPs until the subjects develop deeper confidence and self-efficacy. Then the HCPs typically fade support and are able to effectively provide cost-effective guidance through occasional messages. It is important to note that in no instance will the computer make insulin titration decisions. Decisions will always be made by the subject or the HCPs, and the subject's decisions will always be accompanied by approval/evaluation by the HCPs. The tablet computer simply visualizes the data to make it easier for the subject to make an informed decision.

\section{Statistical analyses}

The primary outcome is the absolute HbAlc level change in 3 months. The secondary outcomes include the percentage reaching the glycemic target of A1c $\leq 7 \%$, the change between patient satisfaction before and after the study, the frequency for hypoglycemia, and the time HCPs and subjects spent on managing the insulin titration. Based on the data published in treat-to-target studies, having $n=20$ in each group across the observed time interval has $88 \%$ power to detect a difference of absolute unit change in HbA1c of $1.0 \%$. A conventional intention-to-treat analysis was used to compare the change in HbA1c level between the groups. A Student's $t$ test was used to compare the mean difference between the intervention and control groups for the measured outcomes.

\section{Control group}

Subjects in the control group received standard care at the clinic in initiating and titrating insulin, with interim faceto-face visits, as well as telephone/fax communication with educators and physicians as dictated by their HCPs. The starting dosage and insulin titration schedule were determined by their HCPs. As part of routine care, the subjects were instructed to contact their HCPs if they experienced any hypoglycemic episode. For the duration of this study, HCPs were requested not to make changes in non-insulin diabetes medications. At the conclusion of 12 weeks, control subjects returned to the center to repeat the HbAlc test, anthropometric measurements, and DTSQ. Rates of hypoglycemia and the frequency of communications were obtained by reviewing the subjects' medical records.

\section{Intervention group}

Subjects met with their HCPs during the initial visit. As in the control group, the HCP team comprised an endocrinologist and certified diabetes educators.

Each subject received a tablet computer at the initial visit. The diabetes management program was preloaded on the 


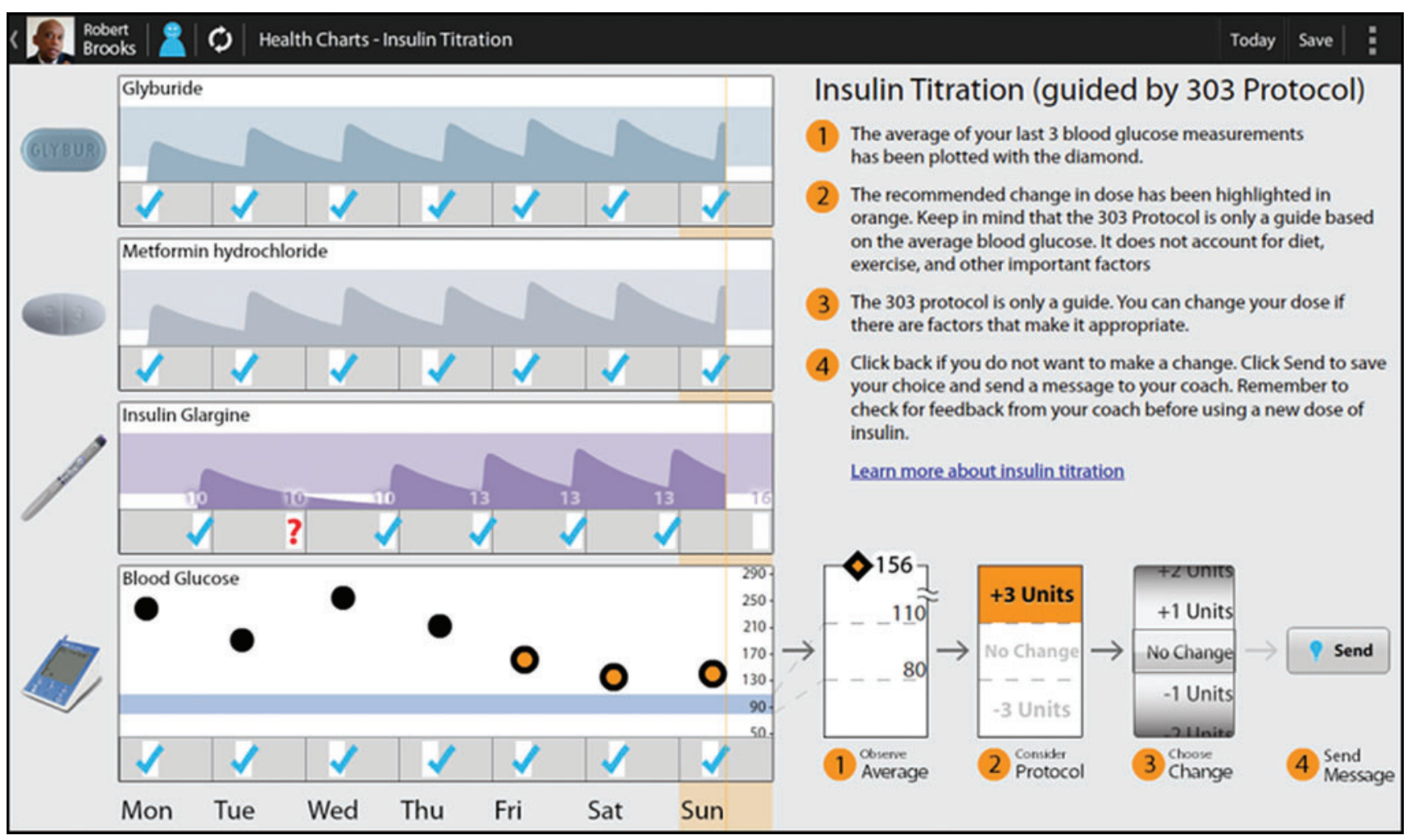

FIG. 2. Insulin titration decision support (PREDICTIVE 303 protocol). On the left side of the screenshot, the charts of the subject's health actions are displayed with each medication adherence and blood glucose adherence event indicated by a check. Pharmacokinetic curves are drawn for medications to highlight subtherapeutic levels from nonadherence, and individual blood glucose readings are plotted. On the right side of the screenshot, personalized decision support for the PREDICTIVE 303 protocol for insulin titration is visualized. Note that the language of the decision support appreciates the likelihood that a healthcare provider considers much more information in making an informed decision than can be accounted for in such a simple algorithm. (The name and photograph used in this example do not belong to any study subject.)

subject's tablet computer with the medication regimen and the initial insulin dose. During the same visit, instructions on using the features and communication tools on the tablet computer were given. A glucose meter that was wirelessly connected to the tablet computer was also given to each subject. Subjects were instructed to perform self-monitoring of blood glucose once a day in the morning with the general goal of achieving a fasting glucose level between 80 and $110 \mathrm{mg} / \mathrm{dL}$. As with the control group, subjects received education on insulin injection according to standard protocol at the center. They were also familiarized with the PREDICTIVE 303 protocol and hypoglycemia treatment guideline. No face-to-face appointments were scheduled during the study period until the end of the study. Subjects had virtual interactions with the HCPs on a regular basis as needed.

As with the control group, no changes in their non-insulin diabetes agents were made during the study. Subjects in the intervention group at the HCP's discretion were generally started on 10 units of basal insulin injection each night. The blood glucose values captured by the wireless glucose meter enabled both the HCPs and the subjects to visualize the glucose pattern on the tablet computer and to use the user interface to communicate desired changes in insulin dosage. The PREDICTIVE 303 protocol served as a guide, not as an absolute rule for insulin titration.
In the beginning the HCPs monitored the glucose values remotely on a daily basis and modeled expert decision making through the communication features on the tablet but gradually intervened less frequently throughout the course of the study to encourage patient self-management. Instead of phone calls, faxes, and face-to-face interactions, the HCPs used a combination of virtual visits (real-time video and voice communication along with shared screen control), asynchronous text messages (secure messages sent in the diabetes management program rather than through traditional short message service channels), or custom features in the software for making collaborative decisions and communicating dosage recommendations.

In addition to collecting the anthropometric measurements, HbA1c measurement, and the DTSQ at the end of the study, subjects underwent an exit interview, which asked about their experience with using the diabetes management system and interaction with their HCPs.

The duration of each virtual visit in the intervention group was electronically tracked by the diabetes management program, as were each of the asynchronous text messages. The total duration of virtual visits and the total number of asynchronous text messages were computed for each subject. Each hypoglycemic reading was also electronically tracked, along with the subject's response as to whether symptoms of hypoglycemia were experienced and what subsequent actions 
were taken (eating a high glycemic snack, rechecking blood glucose, etc.).

\section{Results}

Twenty subjects were randomized to the intervention group, versus 20 to the control group. Five subjects (one from the intervention group and four from the control group) dropped out from the study. Specifically, three failed to show up at the final visit (one from the intervention group and two from the control group), and two opted to participate in a medically supervised weight loss program, which was not part of the study protocol. The baseline characteristics between the control and the intervention groups were comparable in age, weight, height, body mass index, diabetes duration, initial insulin dose, number of non-insulin antidiabetes agents, and DTSQ score (Table 1). In addition, no significant differences were observed for baseline HbAlc $(10.9 \pm 1.2 \%$ in the control group and $10.8 \pm 1.2 \%$ in the intervention group; $P=0.92$ ).

Carrying forward the last $\mathrm{HbA1c}$ result of the subjects who dropped out from the study, the intention-to-treat method was used in analyzing the outcomes. At the end of the study, the intervention group achieved a mean $\mathrm{HbA} 1 \mathrm{c}$ decrease of $3.2 \pm 1.5 \%(P<0.0001)$, with a final HbA1c level of $7.7 \pm 1.6 \%$. In contrast, in the control group, the mean HbA1c decreased by $2.0 \pm 2.0 \% \quad(P=0.0003)$, with a final $\mathrm{HbA} 1 \mathrm{c}$ level of $8.9 \pm 2.2 \%$. The difference in the mean HbA1c decline between the two groups was statistically significant $(P=0.048)$ (Fig. 3). Completers-only analysis showed an HbA1c change from $10.7 \pm 1.2 \%$ to $7.4 \pm 1.2 \%$ in the intervention group $(P<0.0001)$, whereas the control group had an HbA1c change from $10.6 \pm 0.9 \%$ to $8.4 \pm 1.7 \%(P=0.0004)$. There was no statistical difference in the percentage of subjects reaching the HbA1c target of $\leq 7 \%$ between the groups $(P=0.07)$. To further explore the trend in glucose values between the beginning and end of the study in the intervention group, we have analyzed the mean for the first three glucose readings, used for determining the first insulin titration, in Month 1 and compared with the mean of the last three glucose readings in Month 3 used to determine the last insulin titration $(186.8 \pm 56.5 \mathrm{mg} / \mathrm{dL}$ vs. $141.5 \pm 25.7 \mathrm{mg} / \mathrm{dL}: P=0.044)$. We

TAble 1. Baseline Characteristics $(N=40)$

\begin{tabular}{|c|c|c|c|}
\hline & $\begin{array}{c}\text { Control } \\
\text { group }(\mathrm{n}=20)\end{array}$ & $\begin{array}{l}\text { Intervention } \\
\text { group }(\mathrm{n}=20)\end{array}$ & $\mathrm{P}$ value \\
\hline Age (years) & 53.8 & 53.3 & 0.90 \\
\hline Weight (pounds) & 211.1 & 203.9 & 0.64 \\
\hline Height (inches) & 68.7 & 67.4 & 0.27 \\
\hline $\begin{array}{l}\text { Body mass } \\
\text { index }\left(\mathrm{kg} / \mathrm{m}^{2}\right)\end{array}$ & 31.7 & 30.8 & 0.63 \\
\hline $\begin{array}{l}\text { Years from } \\
\text { diagnosis }\end{array}$ & 9.0 & 9.6 & 0.79 \\
\hline HbAlc $(\%)$ & 10.9 & 10.8 & 0.92 \\
\hline $\begin{array}{l}\text { Insulin dosage } \\
\text { (units) }\end{array}$ & 13.3 & 12.0 & 0.34 \\
\hline $\begin{array}{l}\text { Non-insulin } \\
\text { agents }(n)\end{array}$ & 1.8 & 1.9 & 0.49 \\
\hline DTSQ score & 34.3 & 31.9 & 0.41 \\
\hline
\end{tabular}

DTSQ, Diabetes Treatment Satisfaction Questionnaire; HbA1c, hemoglobin A1c. were unable to obtain similar glucose information from the control group.

No significant changes in weight were observed in either group during the study $(-0.48$ pound in the intervention group vs. -0.87 pound in the control group; $P=0.9$ ). The final insulin dose was $24.6 \pm 15.0$ units $(0.27$ units $/ \mathrm{kg})$ in the intervention group and $21.9 \pm 25.0$ units $(0.25$ units $/ \mathrm{kg})$ in the control group $(P=0.69)$. Four subjects in the intervention group and two in the control group reported hypoglycemia during the study. No one required outside assistance in treating hypoglycemia. However, we were only able to obtain hypoglycemic complaints in the control group from subjects who either called following an episode or reported hypoglycemia at the end visit, in contrast to digitally capturing hypoglycemic glucose readings from the intervention group.

Satisfaction with diabetes management was captured through the DTSQ survey. A higher DTSQ score reflects higher satisfaction. The DTSQ score improved significantly, from $31.9 \pm 10.1$ points to $42.0 \pm 3.8$ points $(P=0.001)$ in the intervention group, and only changed from $34.3 \pm 8.5$ points to $36.4 \pm 8.9$ points in the control group $(P=0.1)$. This difference in the change of DTSQ score between the two groups was significant $(P=0.01)$ (Fig. 3).

Face-to-face interaction time for the control group was documented in the medical record, whereas the virtual visit time for the intervention group was captured by the diabetes management program (Table 2). On average, the amount of time required for instructing subjects on how to use the mobile technology was generally less than $1 \mathrm{~h}$ (approximately $40 \mathrm{~min}$ ). The technical instruction was provided by the technical staff on the team, not by HCPs. For the intervention group, there was a mean virtual visit time of 22.5 min per subject, whereas the control group had a mean of $68.8 \mathrm{~min}$ for visit time with clinicians. The average number of messages received and sent in the intervention group was 130.2 per subject in the intervention group. In total, 17 phone calls were conducted between the providers and the subjects in the control group. The mean number of text and video messages sent by subjects in the first, second, and third months decreased over time: $33.4 \pm 25.0,15.3 \pm 16.6$, and 13.6 \pm 12.6 , respectively. Similarly, the mean number of text and video messages received by subjects from HCPs decreased over the 3 months: $39.1 \pm 22.2,13.1 \pm 8$, and $11.8 \pm 10.6$, respectively.

Common themes emerging from the exit interviews revolved around three main areas:

1. Subjects felt that the connectivity with a coach helped them feel less anxious and more motivated to get "back on track when I slip." "I am excited to see what it [glucose reading] is going to say each day." "It's comforting to know that they [clinician coaches] are always there." "I like that it is convenient for me to communicate with my coach." "It [communication with my coach] did not feel intrusive."

2. Subjects felt empowered to make insulin adjustments collaboratively: "I enjoy the power sharing in making decisions on insulin doses." "I feel more equal with the coach in making decision about my health."

3. Subjects felt that they could now make the connection between their glucose reading and their behavior: "I am more conscious of what I eat now." "I didn't know that I felt bad before." "I understand the reasons be- 


\section{Change in HbA1c in 3 months}

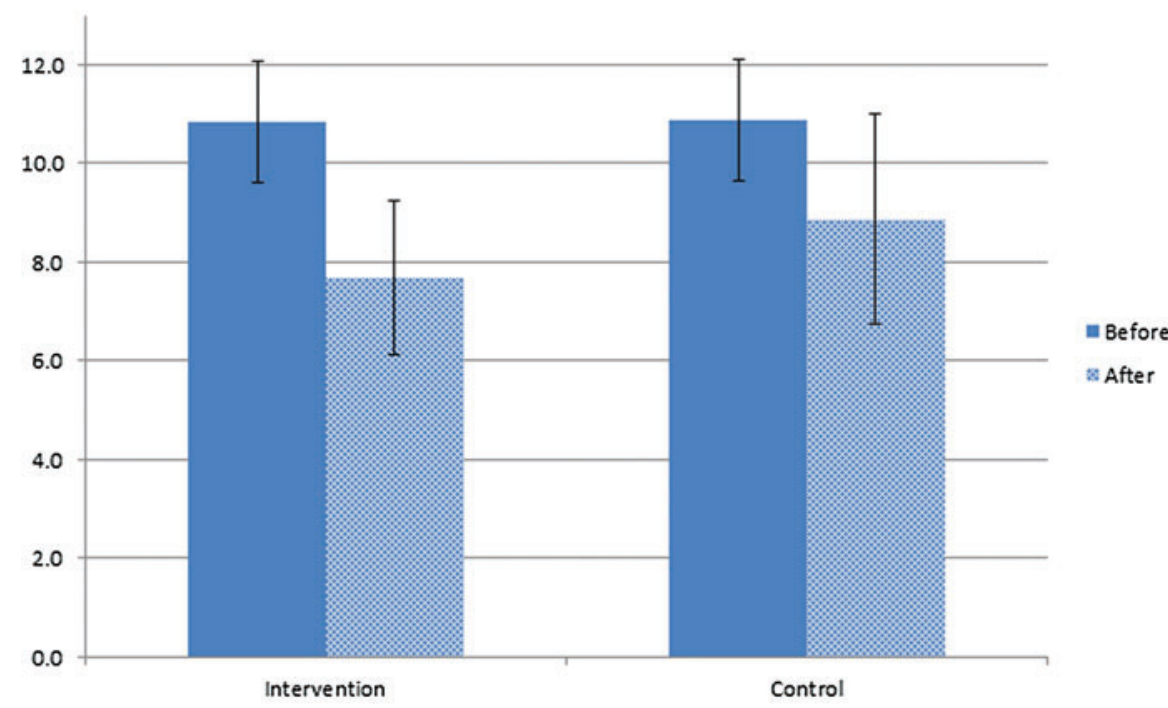

Change in DTSQ Score

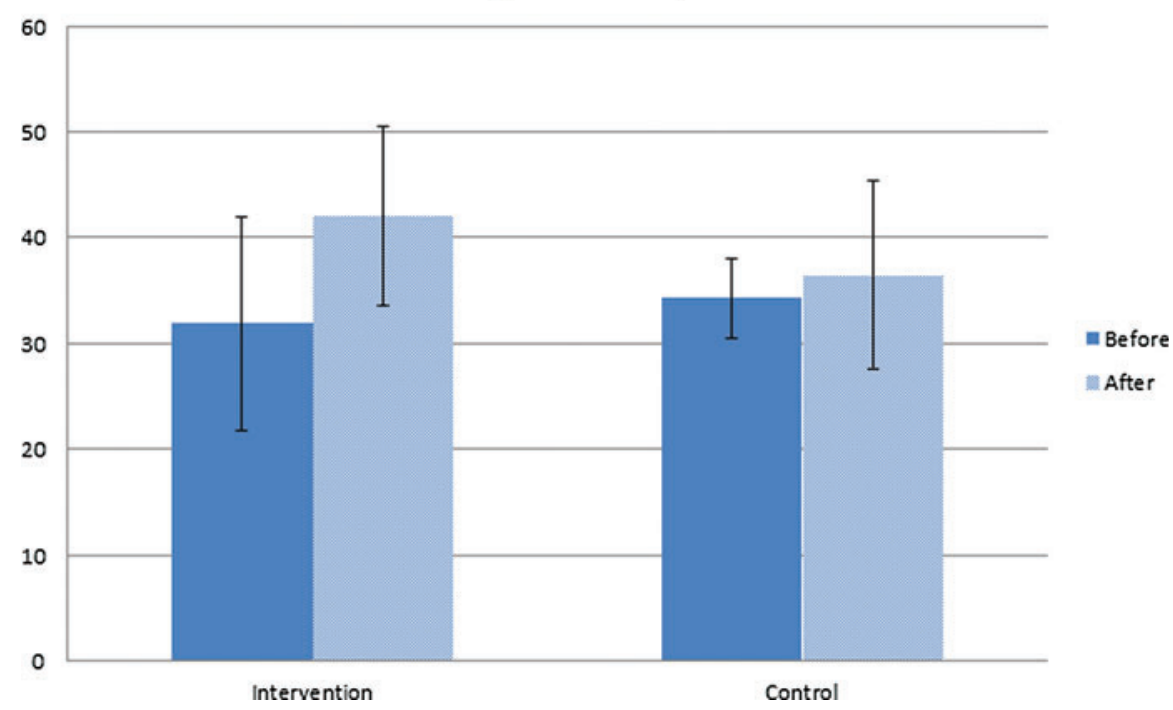

FIG. 3. Changes in (top panel) hemoglobin A1c (HbA1c) and (bottom panel) Diabetes Treatment Satisfaction Questionnaire (DTSQ) score in the intervention group versus the control group over a 3-month period. hind the decision (of changing insulin dose) much better." Subject complaints mainly focused on troubles connecting with the server via their tablet computers, highlighting the importance for a smooth connectivity in the technology design. Another frequent suggestion was a strong desire to connect via their smartphones instead of tablet computers so subjects could access the program without being tied to a WiFi network. Some examples of the text messages sent or received included the following: "Good job, keep up the good work," "Glucose levels have been higher in the morning. Watch your diet," or "My high blood glucose this morning was due to a big snack at bedtime. Will avoid it tonight."

\section{Discussion}

In this feasibility study the use of a cloud-based diabetes management program enabled subjects with type 2 diabetes new to basal insulin therapy to effectively initiate and titrate basal insulin in collaboration with their HCPs. This intervention resulted in a greater decline in $\mathrm{HbA} 1 \mathrm{c}$ level and greater satisfaction of care, while requiring less face-to-face interaction time with the HCPs and without causing more weight gain, severe hypoglycemia, or higher insulin doses than for the controls. The subjects in the intervention group also felt empowered through their connectivity with their HCPs, which helped them better understand the link between their health behavior and its impact on glucose control.

The study substantiated the effectiveness of a cloud-based computer-supported cooperative work model that was grounded in learning science. The recent focus on patient-centricity in health care recognizes that patients are the most underutilized resource in health care. ${ }^{19}$ To enhance patients' selfmanagement skills, according to the theory of situated learning, individuals must learn through solving authentic problems in the context of a personalized social and physical environment rather than from classroom instruction. ${ }^{20}$ We have chosen a 
Table 2. Interaction Time Between Healthcare Providers and Subjects During the Study Period

\begin{tabular}{|c|c|c|c|c|c|c|c|c|}
\hline \multirow[b]{2}{*}{ Group } & \multicolumn{2}{|c|}{$\begin{array}{l}\text { Mean face-to-face } \\
\text { time (min) with }\end{array}$} & \multirow[b]{2}{*}{$\begin{array}{l}\text { Virtual visit } \\
\text { time (min) }\end{array}$} & \multirow{2}{*}{$\begin{array}{c}\text { Mean } \\
\text { messaging } \\
\text { time (min) }\end{array}$} & \multirow{2}{*}{$\begin{array}{l}\text { Mean instruction } \\
\text { time for using } \\
\text { the app (min) }\end{array}$} & \multirow[b]{2}{*}{$\begin{array}{l}\text { Mean phone } \\
\text { time (min) }\end{array}$} & \multicolumn{2}{|c|}{$\begin{array}{l}\text { Total interaction } \\
\text { time }(\text { min })^{\mathrm{b}}\end{array}$} \\
\hline & $\begin{array}{l}M D / \\
N P\end{array}$ & $C D E$ & & & & & $\begin{array}{l}\text { Excluding } \\
\text { app training }\end{array}$ & $\begin{array}{l}\text { Including } \\
\text { app training }\end{array}$ \\
\hline Intervention & NA & NA & 22.5 & 43.4 & 40.0 & NA & 65.9 & 105.9 \\
\hline Control & 20.0 & 48.8 & N/A & NA & NA & 12.8 & 81.6 & 81.6 \\
\hline
\end{tabular}

${ }^{\text {a }}$ Time excluded the initial and exit visits.

${ }^{\mathrm{b}}$ No significant difference measured.

App, application; CDE, certified diabetes educator; MD, medical doctor; NA, not applicable; NP, nurse practitioner.

common challenge in diabetes management to test our hypothesis that short but frequent communications via mobile technologies between a diabetes coach and a patient in the context of daily living can improve glycemic control and facilitate the patient's learning of complex skills in diabetes.

Following the model of cognitive apprenticeship, ${ }^{21}$ subjects in the study went through stages of observation, coaching, and practice. First, the subjects, as learners, repeatedly observed the coach executing the insulin titration process. The subjects then attempted to execute the same process with guidance and help from the coach who provided support in the form of reminders and help that the subjects needed to successfully execute their tasks. Once the subjects were able to master these skills and tasks, the coach reduced her or his participation, providing only limited hints, refinements, and feedback to the learner. In the study the intensity of the interaction between subjects and HCPs, as reflected in the number of text and video messaging, decreased over the study period, further substantiating the impact of cognitive apprenticeship where the time needed to support the subjects decreases over the course of interaction.

Although cognitive apprenticeship has been proven to be effective for learning complex skills, the current model of medical appointments that limits patient-HCP interactions to only medical face-to-face visits makes this approach impractical because of the infrequency of the interactions. In addition, the knowledge transferred during these infrequent face-to-face visits can become out of synchronization with the day-to-day challenges occurring in between medical visits. With the advancement in mobile technology, opportunities exist for HCPs to support patients virtually anytime and anywhere. Furthermore, the real-time application of behavioral sciences, such as closing the behavioral feedback loop by providing decision support in real time, is often the most understated benefits of digital connectivity. Collaborative decision making, enabled through the sharing of data that are transparent to HCPs and patients, has been shown to be successful in empowering patients to manage other chronic illnesses such as hypertension. ${ }^{22}$

The study was conducted in a tertiary center specializing in diabetes care, with the control group receiving high standards of care from diabetes specialists and certified diabetes educators. As a result, the decline in HbA1c level (2\%) in the control group from the study was higher than that reported in studies conducted in primary care settings $(0.6-1.8 \%)$. If the study were to be conducted in general practice, the difference between the intervention and control groups could be more accentuated.
Overall, the HCPs in the intervention group spent one-third of the time on virtual visits compared with the time HCPs spent in face-to-face visits with the subjects in the control group. In addition, the HCPs in the intervention group provided support with text messages, which, on average, between sending and receiving messages, totaled around 130 messages over the course of 12 weeks for each subject (averages about 1.5/day). Given that the nature of the text messages was brief exchanges of encouragement and support and that the more substantive discussions were left to virtual visits, we estimated that it took, on average, about $20 \mathrm{~s}$ to prepare and send or respond to a text message. This would add an additional 43.4 min to the HCPs' time in the intervention group.

To comprehensively assess the total time needed in the intervention group, we also accounted for the time ( $40 \mathrm{~min})$ needed to instruct subjects on the mobile system. From the standpoint of resource management, it is important to note that the instruction was given by technical staff on the research team and was not provided by the HCPs. It is conceivable that such instruction can be built into the mobile system via self-directed learning module or video in the future, thereby minimizing the amount of human resources needed to train the subjects.

The control group as a whole, in addition to spending much more time for face-to-face visits, also utilized a total of 17 phone calls with their HCPs. We estimate that it took about $15 \mathrm{~min}$ for each call, totaling an additional $255 \mathrm{~min}$ for the control group, with a mean of 12.8 min per subject (Table 2). Although it may be more difficult to translate the exact amount of effort HCPs spent on reviewing data and communicating in the intervention group, the time saved by the patients in the intervention group is much easier to appreciate because there was no need to travel to and from the HCP's office for a face-to-face visit and no wait time for these medical appointments.

If time saving can be further substantiated for the HCPs in future studies, the potential exists for busy diabetes specialists to expand their support to more patients through digital technologies. This has enormous implications for managing an increasing number of people with this chronic condition in an environment where the increase in the number of specialists trained to treat this condition is not keeping pace with the rampant rise in the prevalence of diabetes. Lastly, it is also important to note that the time spent coaching and supporting subjects on the use of the technology is not trivial. Future designs must take this factor into consideration. 
Interestingly, the blood glucose levels for some subjects in the intervention group improved just a few days after insulin initiation, possibly pointing to the insulin's effectiveness of targeting glucose toxicity. ${ }^{23}$ The immediate glycemic improvement might have also have resulted from behavioral changes as subjects were motivated by accountability to their coach.

The age of the subjects in the intervention group ranged from 23 to 80 years. With proper support and training, the senior subjects were able to operate and use the data from the tablet computer. As the senior citizens in our society are increasingly adopting mobile technology to assist with their health, ${ }^{24}$ software designers and HCPs need to work together to design features and user interfaces suitable for this population. This becomes even more relevant as the prevalence of diabetes increases in our aging population, and more people will require insulin treatment to control their condition.

The evidence for the effectiveness of mobile health applications is growing in areas of diabetes management and weight loss and is applicable to diverse populations. ${ }^{25-28}$ Although the current face-to-face care model has been the standard of care for decades, it is primarily clinic-based and therefore does not scale as an information technology solution might. If the technology-supported apprenticeship model could allow medical personnel to outperform themselves using virtual tools, then not only could they achieve better results for their patients, but they could also provide their services to many more people who require it. As an example, the Mobile Diabetes Intervention Study is a clinical trial that involved 163 patients randomized to usual care and mobile/internet coaching. ${ }^{29}$ Subjects received automated, real-time educational and behavioral messaging in response to individually analyzed blood glucose values, diabetes medications, and lifestyle behaviors communicated by mobile phone. The mean declines in HbAlc levels after 12 months were $1.9 \%$ in the intervention group and $0.7 \%$ in the usual care group $(P<0.001)$. In a time-restrained environment and with the HCP workforce already in distress, reducing HCPs' time and burden in supporting patients through mobile health will be critically important to gain adoption by physicians. Furthermore, future studies will need to demonstrate cost-effectiveness for payers to reimburse for virtual visits.

There are several limitations in the study. A larger sample size is needed to confirm our findings. A more thorough capture of the data in the control group is necessary to enable comparison of important parameters such as frequency of hypoglycemia and medication adherence. Furthermore, a more thorough capture of the amount of time spent on paperwork versus virtual documentation would be valuable to better illustrate time investment.

In summary, diabetes is a chronic illness where patients need to have the necessary support and training in between medical visits to gain the skills and knowledge for diabetes self-management. Mobile health technologies provide the platform for real-time connectivity, decision support for both patients and HCPs, and collaborative decision making. When combined with behavioral and learning science, mobile health technologies have the potential to empower patients and increase the efficiency and effectiveness of HCPs to care for their patients.

\section{Acknowledgments}

We would like to thank Dr. Aldo Rossini for his lifelong commitment to improving patient care. His tireless passion inspires us to provide better care for our patients.

\section{Author Disclosure Statement}

John Moore is now the CEO of Twine Health, a company that creates software for chronic disease management.

\section{References}

1. Peters AL, Legorreta AP, Ossorio RC, et al.: Quality of outpatient care provided to diabetic patients. A health maintenance organization experience. Diabetes Care 1996;19: 601-606.

2. World Health Organization: Global Status Report on Noncommunicable Diseases. 2010. www.who.int/nmh/publications/ ncd_report_full_en.pdf (accessed February 7, 2015).

3. Inzucchi SE, Bergenstal RM, Buse JB, et al.: Management of hyperglycemia in type 2 diabetes, 2015: a patient-centered approach: update to a position statement of the American Diabetes Association and the European Association for the Study of Diabetes. Diabetes Care 2015;38:140-149.

4. American Diabetes Association: (7) Approaches to glycemic treatment. Diabetes Care 2015;38(Suppl):S41-S48.

5. Inzucchi SE, Bergenstal RM, Buse JB, et al.: Management of hyperglycemia in type 2 diabetes: a patient-centered approach: position statement of the American Diabetes Association (ADA) and the European Association for the Study of Diabetes (EASD). Diabetes Care 2012;35:1364-1379.

6. Rosenstock J, Davies M, Home PD, et al.: A randomised, 52week, treat-to-target trial comparing insulin detemir with insulin glargine when administered as add-on to glucoselowering drugs in insulin-naive people with type 2 diabetes. Diabetologia 2008;51:408-416.

7. Riddle MC, Rosenstock J, Gerich J, et al.: The treat-totarget trial: randomized addition of glargine or human NPH insulin to oral therapy of type 2 diabetic patients. Diabetes Care 2003;26:3080-3086.

8. Swinnen SG, Dain MP, Aronson R, et al.: A 24-week, randomized, treat-to-target trial comparing initiation of insulin glargine once-daily with insulin detemir twice-daily in patients with type 2 diabetes inadequately controlled on oral glucose-lowering drugs. Diabetes Care 2010;33:1176-1178.

9. Meneghini L, Koenen C, Weng W, et al.: The usage of a simplified self-titration dosing guideline (303 Algorithm) for insulin detemir in patients with type 2 diabetes-results of the randomized, controlled PREDICTIVE 303 study. Diabetes Obes Metab 2007;9:902-913.

10. Budnitz DS, Lovegrove MC, Shehab N, et al.: Emergency hospitalizations for adverse drug events in older Americans. N Engl J Med 2011;365:2002-2012.

11. Weinstock RS, Teresi JA, Goland R, et al.: Glycemic control and health disparities in older ethnically diverse underserved adults with diabetes: five-year results from the Informatics for Diabetes Education and Telemedicine (IDEATel) study. Diabetes Care 2011;34:274-279.

12. Charpentier G, Benhamou PY, Dardari D, et al.: The Diabeo software enabling individualized insulin dose adjustments combined with telemedicine support improves HbA1c in poorly controlled type 1 diabetic patients: a 6-month, randomized, open-label, parallel-group, multicenter trial (TeleDiab 1 Study). Diabetes Care 2011;34: 533-539. 
13. Davis RM, Hitch AD, Salaam MM, et al.: TeleHealth improves diabetes self-management in an underserved community: diabetes TeleCare. Diabetes Care 2010;33:1712-1717.

14. Turner J, Larsen M, Tarassenko L, et al.: Implementation of telehealth support for patients with type 2 diabetes using insulin treatment: an exploratory study. Inform Prim Care 2009; 17:47-53.

15. Joslin Diabetes Center: Joslin Clinical Guideline for Adults with Diabetes. https://www.joslin.org/docs/Adult_guideline_ -update_thru_10-23-14_2.pdf (accessed December 15, 2014).

16. Cryer PE, Davis SN, Shamoon H: Hypoglycemia in diabetes. Diabetes Care 2003;26:1902-1912.

17. Hermansen K, Davies M, Derezinski T, et al.: A 26-week, randomized, parallel, treat-to-target trial comparing insulin detemir with NPH insulin as add-on therapy to oral glucoselowering drugs in insulin-naive people with type 2 diabetes. Diabetes Care 2006;29:1269-1274.

18. Moore J: A new wave of patient-centered care: apprenticeship in the management of chronic disease. J Clin Outcomes Manage 2012;19:293-300.

19. Kraschnewski JL, Gabbay RA: Role of health information technologies in the Patient-centered Medical Home. J Diabetes Sci Technol 2013;7:1376-1385.

20. Brown J, Collins A, Duguid P: Situated cognition and the culture of learning. Educ Res 1989;18:32-42.

21. Collins A, BBN Laboratories, Brown JS, Newman SE, Xerox Palo Alto Research Center: Cognitive Apprenticeship: Teaching the Craft of Reading, Writing, and Mathematics. Technical Report No. 403. Champaign, IL: University of Illinois at Urbana-Champaign, 1987.

22. Moore J, Marshall M, Judge D, et al.: Technology-supported apprenticeship in the management of hypertension-a randomized controlled trial. J Clin Outcomes Manag 2014;21: $110-122$.
23. Campbell RK, White JR: Insulin therapy in type 2 diabetes. J Am Pharm Assoc (Wash) 2002;42:602-611.

24. Barrett L: Health and Caregiving Among 50+: Ownership, Use and Interest in Mobile Technology. http://assets.aarp.org/ rgcenter/general/health-caregiving-mobile-technology.pdf (accessed March 10, 2015).

25. Arsand E, Frøisland DH, Skrøvseth SO, et al.: Mobile health applications to assist patients with diabetes: lessons learned and design implications. J Diabetes Sci Technol 2012;6:1197-1206.

26. Quinn CC, Clough SS, Minor JM, et al.: WellDoc mobile diabetes management randomized controlled trial: change in clinical and behavioral outcomes and patient and physician satisfaction. Diabetes Technol Ther 2008;10:160-168.

27. Gerber BS, Stolley MR, Thompson AL, et al: Mobile phone text messaging to promote healthy behaviors and weight loss maintenance: a feasibility study. Health Informatics J 2009;15: $17-25$.

28. Tate DF, Wing RR, Winett RA: Using Internet technology to deliver a behavioral weight loss program. JAMA 2001;285: 1172-1177.

29. Quinn CC, Shardell MD, Terrin ML, et al.: Clusterrandomized trial of a mobile phone personalized behavioral intervention for blood glucose control. Diabetes Care 2011; 34:1934-1942.

Address correspondence to:

William C. Hsu, MD

Joslin Diabetes Center

Harvard Medical School

One Joslin Place

Boston, MA 02215

E-mail: william.hsu@joslin.harvard.edu 